\title{
Correction to: Improved Mechanical Properties of a Quenched and Partitioned Medium-Carbon Bainitic Steel by Control of Bainitic Isothermal Transformation
}

\author{
Qiangguo Li, Yanan Zhang, Wandong Li, Xuefei Huang, and Weigang Huang
}

\section{Correction to: \\ JMEPEG (2020) 29:32-41 \\ https://doi.org/10.1007/s11665-020-04554-X}

In the originally published article, Weigang Huang should have been listed as a corresponding author, along with Qiangguo Li. The authors regret the oversight, and the correction is made with the agreement of all authors. Weigang Huang can be contacted at huangwg56@163.com.

Publisher's Note Springer Nature remains neutral with regard to jurisdictional claims in published maps and institutional affiliations.

The original article can be found online at https://doi.org/10.1007/ s11665-020-04554-x.

Qiangguo Li, College of Architecture and Environment, Sichuan University, Chengdu 610065, People's Republic of China; and College of Materials Science and Engineering, Sichuan University, Chengdu 610065, People's Republic of China; Yanan Zhang, School of Materials Engineering, Xi'an Aeronautical University, Xi'an 710077, People's Republic of China; Wandong Li, Chengde Petroleum College, Chengde 067000, People's Republic of China; and Xuefei Huang and Weigang Huang, College of Materials Science and Engineering, Sichuan University, Chengdu 610065, People's Republic of China. Contact e-mails: LeeValiant@163.com and huangwg56@163.com. 